\title{
24-Hour blood pressure response to lower dose (30 mg) fimasartan in Korean patients with mild to moderate essential hypertension
}

\author{
Hae-Young Lee ${ }^{1}$, Cheol-Ho Kim², Jae-Kwan Song ${ }^{3}$, Shung Chull Chae ${ }^{4}$, Myung Ho Jeong 5 , \\ Dong-Soo Kim ${ }^{6}$, and Byung-Hee $\mathrm{Oh}^{1}$
}

Department of Internal Medicine, ${ }^{1}$ Seoul National University Hospital, Seoul; ${ }^{2}$ Seoul National University Bundang Hospital, Seongnam; ${ }^{3}$ Asan Medical Center, University of Ulsan College of Medicine, Seoul; ${ }^{4}$ Kyungpook National University Hospital, Daegu; ${ }^{5}$ The Heart Center of Chonnam National University Hospital, Gwangju; ${ }^{6}$ Inje University Busan Paik Hospital, Busan, Korea

Received: March 20, 2016

Revised : July 6, 2016

Accepted: August 23, 2016

\section{Correspondence to}

Byung-Hee Oh, M.D.

Department of Internal

Medicine, Seoul National

University College of Medicine,

101 Daehak-ro, Jongno-gu, Seoul 03080, Korea

Tel: $+82-2-2072-3345$

Fax: +82-2-3674-0805

E-mail: ohbhmed@snu.ac.kr
Background/Aims: Fimasartan is an angiotensin type 1 receptor blocker (ARB) which has comparable efficacy and tolerability with other ARBs. The aim of this study was to evaluate 24-hour blood pressure (BP) lowering efficacy and the tolerability of the low dose fimasartan compared with valsartan in patients with mild to moderate hypertension.

Methods: This study was a phase II, prospective, multicenter, randomized, double-blind, parallel-grouped trial. A total of 75 hypertensive patients, whose mean ambulatory $\mathrm{BP}$ monitoring values were $\geq 135 / 85 \mathrm{mmHg}$, were randomized to either fimasartan $30 \mathrm{mg}$ or valsartan $80 \mathrm{mg}$ daily. The primary efficacy endpoint was the change in the mean 24-hour systolic BP (SBP) values from the baseline and at the week 8 . Secondary endpoints included the change in the mean 24-hour diastolic BP values, the daytime and the nighttime mean BP values at week 8 , the trough-to-peak $(\mathrm{T} / \mathrm{P})$ ratio and the smoothness index.

Results: At week 8, the mean 24-hour SBP values significantly decreased in both groups; $-10.5 \pm 11.9 \mathrm{mmHg}(p<0.0001)$ in the fimasartan group and $-5.5 \pm 11.6$ $\mathrm{mmHg}(p=0.0307)$ in the valsartan group. The difference between two groups was $4.3 \pm 2.9 \mathrm{mmHg}$ but there was no statistical significance $(p=0.1392)$. The global $\mathrm{T} / \mathrm{P}$ ratio in the fimasartan $30 \mathrm{mg}$ groups were 0.48 and 0.40 in the valsartan $80 \mathrm{mg}$ group, respectively $(p=0.3411)$. The most frequent adverse events (AEs) were acute pharyngitis and there were no cases of severe AEs.

Conclusions: In mild-to-moderate hypertensive patients, low dose (30 mg) fimasartan showed comparable 24-hour BP lowering efficacy compared with valsartan (80 mg). There was no difference in tolerability between two groups.

Keywords: Hypertension; Angiotensin II type 1 receptor blockers; Blood pressure monitoring, ambulatory

\section{INTRODUCTION}

Hypertension is the most common cause of cardiovascular disease [1]. Blood pressure (BP) control by antihypertensive medication is effective strategy to reduce cardiovascular morbidity and mortality $[2,3]$. Among the different classes of antihypertensive drugs, angiotensin type 1 receptor blockers (ARBs), which regulate the renin-angiotensin-aldosterone system by restricting the action of angiotensin II, are recommended as a first-line 
therapy in all guidelines [4-8].

Fimasartan, developed by Boryung Pharmaceutical Co. Ltd., Seoul, Korea, is the eighth ARB out of nine currently available ARBs $[9,10]$. Fimasartan at the range of 30 to $120 \mathrm{mg}$ once daily showed an effective BP lowering effect in hypertensive patients [11-14]. Also in large population observation study, fimasartan showed excellent safety profile $[15,16]$. Several pharmacokinetic/ pharmacodynamic studies were performed to evaluate the possible drug or food interactions [17-20] and the drug metabolism in the elderly population and in hepatic dysfunction $[21,22]$. In patients with moderate hepatic impairments, the bioavailability of fimasartan was 5 -fold higher than that of healthy controls $[20,21]$. The co-administration of fimasartan with amlodipine, hydrochlorothiazide, or digoxin did not show any significant interactions [23-25]. Conversely, fimasartan raised plasma atorvastatin concentrations [20].

Previously, fimasartan $60 \mathrm{mg}$ was the minimum permitted dose for hypertension treatment. However, in real world situation, half tablet ( $30 \mathrm{mg}$ ) of fimasartan was often used and was suggested to have sufficient BP lowering efficacy. Also, fimasartan $30 \mathrm{mg}$ showed superior office BP lowering efficacy than valsartan $80 \mathrm{mg}$ in patients with mild to moderate hypertension [26]. With this background, this study was conducted to evaluate whether once daily administration of the low dose 30 $\mathrm{mg}$ ) fimasartan is able to maintain BP lowering toward the end of the dosing interval. Ambulatory blood pressure monitor (ABPM) was performed to evaluate 24 -hour BP lowering efficacy of the low dose fimasartan (30 mg once daily) compared with valsartan (8o mg once daily) in patients with mild to moderate hypertension.

\section{METHODS}

\section{Patients}

Male or non-childbearing female patients aged 20 to 70 with mild to moderate hypertension whose mean 24hour ABPM values $\geq 135 / 85 \mathrm{mmHg}$ were eligible for the study. For office BP measurement, patients should be seated at least for 5 minutes and refrain from smoking or ingesting caffeine during the 30 minutes prior to the measurement. Patients were excluded from the study if they met any of the following exclusion criteria:(1) severe hypertension defined as mean sitting diastolic blood pressure $(\mathrm{SiDBP}) \geq 110 \mathrm{mmHg}$ or mean sitting systolic blood pressure $(\mathrm{SiSBP}) \geq 180 \mathrm{mmHg}$ at the screening, baseline and randomization visits; (2) a change in SiDBP $\geq 10 \mathrm{mmHg}$ or a change in SiSBP $\geq 20 \mathrm{mmHg}$ measured in the same arm at the screening visit; (3) secondary hypertension including pheochromocytoma, renal artery stenosis, and functional adrenal adenoma; (4) symptomatic orthostatic hypotension; (5) uncontrolled diabetes before the screening visit (glycosylated hemoglobin > $9 \%)$, current treatment with insulin, or a change in oral hypoglycemic agent dosage/usage within the 12 weeks before the screening visit; (6) history of myocardial infarction or coronary arterial disease, clinically significant congestive heart failure or valvular heart disease identified within the past six months; and (7) serum creatinine $\geq 1.5$ times upper normal limit $(<1.40 \mathrm{mg} / \mathrm{dL})$, aspartate aminotransferase or alanine aminotransferase $\geq 2$ times upper normal limit (< $40 \mathrm{IU} / \mathrm{L})$, and other clinically significant abnormal laboratory test results.

\section{Study design}

This study was a phase II, prospective, multicenter, randomized, double-blind, parallel-grouped trial to compare 24-hour BP lowering efficacy and the tolerability of the low-dose fimasartan and valsartan in patients with mild to moderate essential hypertension. All of the study protocols were reviewed and approved by the Institutional Review Board of each participating hospital prior to any patient enrollment (IRB No. 1102-072-352). In addition, the study was conducted in accordance with the current Good Clinical Practices and other applicable laws and regulatory requirements in Korea. This study was registered in Clinicaltrials.gov (NCTo1878201).

Subjects went through a screening period after voluntary agreement to participate in the study including a 7-day washout period for those patients who had been taking other medication. After 2 weeks' placebo run-in, the eligible hypertensive patients, whose mean ABPM values were $\geq 135 / 85 \mathrm{mmHg}$, were randomized to either fimasartan $30 \mathrm{mg}$ daily or valsartan $80 \mathrm{mg}$ daily. Patients were instructed to orally take the assigned drug once daily at the same time after a meal in the morning while they came to the clinic without taking the drug when study procedures were scheduled at weeks 0,4 , and 8. During these study visits, BP measurements, physical 
examinations, clinical laboratory tests (i.e., hematology, blood chemistry, and urinalysis), and 12-lead electrocardiogram (ECG) were performed.

Compliance was assessed by investigators based on the rate of return and amount of administration of investigational products. Clinical pharmacists recorded the amount of returned product when a subject delivered the remaining product to pharmacist. Compliance was assessed as below: compliance (\%) = (total number of administered tablets / total number of tablets to be administered according to the protocol) $\times 100$. The study design was summarized in Fig. 1.

\section{4-Hour ambulatory blood pressure monitoring}

We used the same protocol as previous ABPM study with fimasartan 20 to $180 \mathrm{mg}[11,12]$. Every center used same ABPM device of HEM-7080IT (equivalent to 705IT, Omron, Kyoto, Japan). With patients reporting at the clinic in the morning, ABPM started and continued for the subsequent 24 hours. During ABPM, patients were instructed to record daily events and were discouraged from engaging in strenuous physical activities. Patients were also informed in advance of the possible problems related to ABPM and instructed how to stop ABPM when they were occurred. Ambulatory BP was recorded every 20 minutes from 7:00 AM to 10:59 PM (daytime) and every 30 minutes from 11:00 PM to 6:59 AM the next day (nighttime). BP recordings were accepted only if they spanned the full 24 hours and at least $80 \%$ of the expected recordings were recorded. Readings of SBP < 70 or $>250 \mathrm{mmHg}$ and $\mathrm{DBP}<40$ or $>150 \mathrm{mmHg}$ were discarded and daytime BPs were averaged over a 1-hour period. ABPs were then synchronized (i.e., matched by measurement time) in each patient and 8-week values

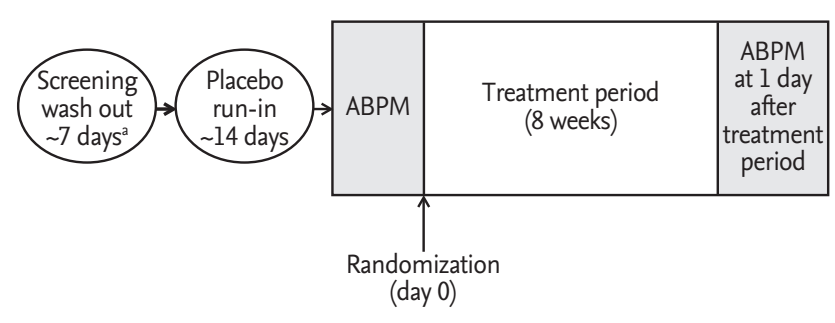

Figure 1. Study design. ABPM, ambulatory blood pressure monitor. ${ }^{\text {a }}$ Previous anti-hypertensive medication users only. were subtracted from baseline values to determine 24hour, daytime, and nighttime mean changes.

The trough-to-peak (T/P) ratio was calculated to evaluate the sustainability of $\mathrm{BP}$ reduction toward the end of the dosing interval. The trough was defined as the ambulatory BP reduction over the last 2 hours of the dosing interval (i.e., 23 to 24 hours post-dose) [27]. The peak effect was defined as the maximal decrease in ambulatory BP from baseline over any 1-hour period after drug administration using 2-hour moving averages [27]. ABPM data were then matched by measurement time in each patient, and values at 8 weeks of treatment were subtracted from the baseline values to determine the trough and peak effects. From these effects, global T/P ratio was calculated from all patients for a single effect profile (global T/P ratio), as previously recommended $[28,29]$.

The smoothness index (SI) was also calculated to evaluate all treatment effects over the full 24-hour period [30]. SI was calculated as the ratio of the mean of hourly BP changes to their standard deviation using the measurement time-matched ABPM data. A greater SI denotes lesser variability of $\mathrm{BP}$ reduction by treatment over the full dosing interval, although there is no reference value for SI [31].

\section{End points}

The primary efficacy endpoint was the change in the mean 24-hour SBP values from the baseline and at the week 8. Secondary endpoints included the change in the 24-hour mean DBP values from the baseline at the week 8 , the daytime and the nighttime mean $\mathrm{BP}$ values at week 8 , the T/P ratio and the SI of each drugs. T/P ratio analysis was performed individually and globally as previously recommended in the latter case of which all patients were combined to come up with a single effect profile, from which global estimates were determined $[12,28]$.

Safety endpoints were treatment-emergent adverse events (TEAEs), which were defined as any untoward events not present prior to the administration of the study drug or already present but worsened in either intensity or frequency during treatment. TEAEs included abnormalities found in clinical laboratory tests, physical examinations, and ECG readings. 


\section{Sample size and statistical analysis}

Since this study was initiated as an exploratory study in order to respond the practical need from the clinical field, we enrolled 35 patients in each group which was the minimum regulatory requirements in Korean governmental regulation for addition of the new dosage form. With an anticipated dropout rate of 10\%, a total of 68 patients were required to fulfill the enrollment of 34 patients in two groups.

Efficacy data was evaluated mainly in full analysis set (FAS) group and also in the per-protocol (PP) group. The FAS population consisted of the subjects who had the baseline $\mathrm{BP}$ and $\geq$ one post-randomization $\mathrm{BP}$ values and who had taken $\geq$ one dose of study drug. The PP population consisted of all subjects within the FAS population who did not commit any major protocol violation and drop-out that would likely to affect the efficacy outcomes. The safety set population consisted of the group of subjects who received investigational products at least once or more after randomization.

Changes from the baseline in the mean 24-hour SBP/ $\mathrm{DBP}$, the daytime and the nighttime SBP/DBP at 8 weeks were analyzed by paired $t$ test in each treatment group. The between-group differences were analyzed by analysis of covariance model using site and baseline as covariates [13]. Nonparametric tests such as the Wilcoxon rank sum test were also used when deemed necessary. The proportion of patients experiencing adverse events (AEs) and its $95 \%$ confidence interval were also presented by the treatment group and a chi-square test or Fisher exact test was performed for the between-group difference. SAS version 9.3 (SAS Institute, Inc., Cary, NC, USA) was used for statistical analysis.

\section{RESULTS}

\section{Patient recruitment and baseline characteristics}

In six institutions, 75 patients among 138 patients, who were screened and fulfilled the selection criteria, were randomly allocated into two treatment groups. During placebo run-in period, two patients withdrew consent and four patients were lost to follow-up. Among 75 patients who were randomized and took a single dose of the study drug, 67 patients completed the study. The reasons for discontinuation were as followed; withdraw-

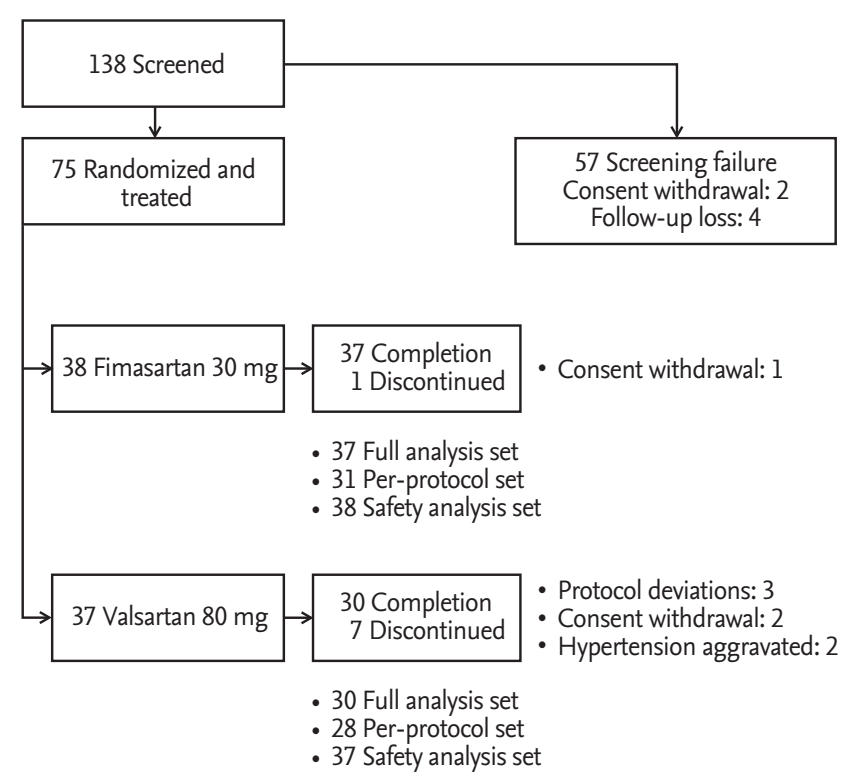

Figure 2. Patients recruitment and flow.

al of consent (three patients), protocol deviations (three patients), and investigators' decision of safety concern for BP elevation (two patients). Patient recruitment and flow were summarized in Fig. 2.

Two treatment groups were comparable for all baseline demographic and clinical characteristics except higher BMI in the fimasartan group (Table 1). The mean \pm SE age was $57.1 \pm 7.6$ years old and the proportion of male patients was 70.2\%. Mean baseline office SBP/DBP were $147.4 \pm 12.6 / 90.4 \pm 8.6 \mathrm{mmHg}$. The $73.1 \%$ of patients had been previously treated with anti-hypertensive agents, most frequently with ARBs (37.3\%), calcium channel blockers (32.8\%), and $\beta$-blockers (7.5\%). There was no significant difference in previous anti-hypertensive medication history between two groups (Table 1).

\section{Efficacy outcomes}

At week 8, the mean 24-hour SBP significantly decreased from the baseline in both groups; $-10.5 \pm 11.9 \mathrm{mmHg}$ in the fimasartan group $(p<0.0001)$ (Fig. $3 \mathrm{~A})$ and $-5.5 \pm$ $11.6 \mathrm{mmHg}$ in the valsartan group $(p=0.0307$ ) (Fig. $3 \mathrm{~B}$ ). The difference between two groups (least square mean \pm $\mathrm{SE}$ ) was $4.3 \pm 2.9 \mathrm{mmHg}$ but there was no statistical significance $(p=0.1392$ ) (Fig. 3 C). PP group showed similar results; $-11.1 \pm 12.6 \mathrm{mmHg}$ in the fimasartan group $(p$ $<0.0001)$ and $-6.1 \pm 11.7 \mathrm{mmHg}$ in the valsartan group $(p=0.0108)$, but there was no statistical significant dif- 
ference between two groups, either $(p=0.1552)$ (Table 2). The mean 24-hour DBP also significantly decreased from the baseline in both groups; $-5.8 \pm 6.4 \mathrm{mmHg}(p<$ $0.0001)$ in the fimasartan group and $-5.0 \pm 7.3 \mathrm{mmHg}(p$ $=0.0008$ ) in the valsartan group, but there was no statistical significant difference between two groups, either $(p$ $=0.9732$ ).

After 8 weeks' treatment, both fimasartan $30 \mathrm{mg}$ and valsartan $80 \mathrm{mg}$ significantly reduced the daytime, and the nighttime mean SBP by $-8.0 \pm 12.1 \mathrm{mmHg}$ and -4.1 $\pm 12.1 \mathrm{mmHg}$ and $-15.7 \pm 14.9 \mathrm{mmHg}$ and $-8.0 \pm 15.3$ $\mathrm{mmHg}$, respectively (Table 3).

The changes of the mean 24-hour, the daytime and the nighttime BPs from the baseline at week 8 were illustrated in Fig. 4.

Fimasartan $30 \mathrm{mg}$ daily was associated with the similar BP reduction toward the end of the dosing interval compared with valsartan $80 \mathrm{mg}$ daily, which resulted in statistically insignificant difference of $\mathrm{T} / \mathrm{P}$ ratio. The global $\mathrm{T} / \mathrm{P}$ ratios for ambulatory SBP and DBP were 0.48 and 0.34 in the fimasartan group and 0.40 and 0.52 in the valsartan group ( $p=0.3411$ for SBP, $p=0.7288$ for DBP). The SIs for ambulatory SBP and DBP were 0.86 and 0.63 in the fimasartan group and 0.51 and 0.58 in the valsartan group ( $p=2538$ for SBP, $p=0.7885$ for DBP) (Table 4 ).

\section{Tolerability}

Both group showed excellent compliance rate of $96.6 \%$ $\pm 4.0 \%$ (96.6 \pm 3.7 vs. $96.2 \pm 4.3, p=0.8418)$. Although we collected all the activity and the event during ABPM measurement, there were no remarkable events reported. The proportions of patients experiencing $\geq 1$ TEAE were comparable between two groups; four patients (five events, $10.5 \%$ ) in the fimasartan group and six patients (eight events, $16.2 \%)$ in the valsartan group ( $p=0.5161)$. No serious TEAEs were reported, and acute pharyngi-

Table 1. Baseline demographic and clinical characteristics

\begin{tabular}{|c|c|c|c|c|}
\hline Characteristic & Fimasartan $30 \mathrm{mg}(\mathrm{n}=37)$ & Valsartan $80 \mathrm{mg}(\mathrm{n}=30)$ & $\operatorname{Total}(\mathrm{n}=67)$ & $p$ value \\
\hline Age, yr & $57.1 \pm 7.9$ & $57 \cdot 0 \pm 7 \cdot 4$ & $57.1 \pm 7.6$ & 0.957 \\
\hline Male sex, \% & 73 & 67 & 70 & 0.575 \\
\hline Height, cm & $165.6 \pm 8.1$ & $168.0 \pm 8.2$ & $166.7 \pm 8.2$ & 0.228 \\
\hline Weight, kg & $71.4 \pm 11.1$ & $68.2 \pm 10.7$ & $69.9 \pm 11.0$ & 0.238 \\
\hline Body mass index, $\mathrm{kg} / \mathrm{m}^{2}$ & $25.95 \pm 2.87$ & $24.07 \pm 2.66$ & $25.11 \pm 2.91$ & 0.008 \\
\hline SiDBP, mmHg & $91.8 \pm 7.8$ & $88.6 \pm 9.3$ & $90.4 \pm 8.6$ & 0.126 \\
\hline SiSBP, mmHg & $148.4 \pm 13.5$ & $146.3 \pm 11.5$ & $147 \cdot 4 \pm 12.6$ & 0.865 \\
\hline Pulse rate, BPM & $70.6 \pm 10.0$ & $70.7 \pm 7.6$ & $70.7 \pm 8.9$ & 0.973 \\
\hline Smoking, \% & $13 \cdot 51$ & 20.00 & 16.42 & 0.463 \\
\hline Drinker, \% & $59 \cdot 46$ & $63 \cdot 33$ & 61.19 & 0.746 \\
\hline Previous antihypertensive medication & $29(78.4)$ & $20(66.7)$ & $49(73.1)$ & 0.282 \\
\hline RAS inhibitors & $14(37.8)$ & $11(36.7)$ & $25(37 \cdot 3)$ & \\
\hline Calcium antagonists & $14(37.8)$ & $8(26.7)$ & $22(32.8)$ & \\
\hline$\beta$-Blockers & $4(10.8)$ & $1(3.3)$ & $5(7 \cdot 5)$ & \\
\hline Diuretics & $1(2.7)$ & o & $1(1.5)$ & \\
\hline Other medication & & & & 0.053 \\
\hline Statins & 10 & 5 & 15 & \\
\hline Antithrombotic agents & 9 & 2 & 11 & \\
\hline Antidiabetic agents & 3 & 1 & 4 & \\
\hline Others & 3 & 4 & 7 & \\
\hline
\end{tabular}

Values are presented as mean \pm SD or number (\%). Difference between treatment groups was analyzed by two sample $t$ test or chi-square test.

SiDBP, sitting diastolic blood pressure; SiSBP, sitting systolic blood pressure; BPM, beat per minute; RAS, renin-angiotensin system. 


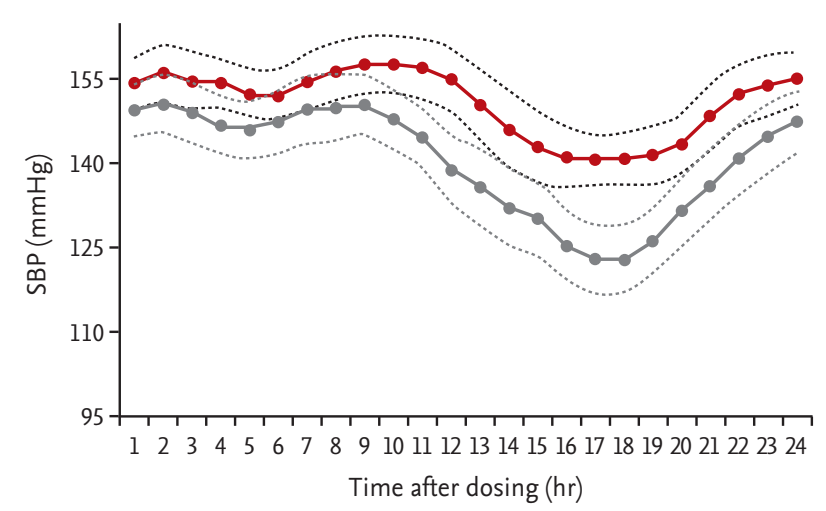

A

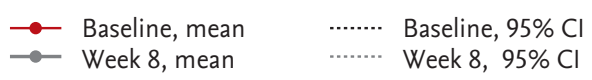

Time after dosing $(\mathrm{hr})$

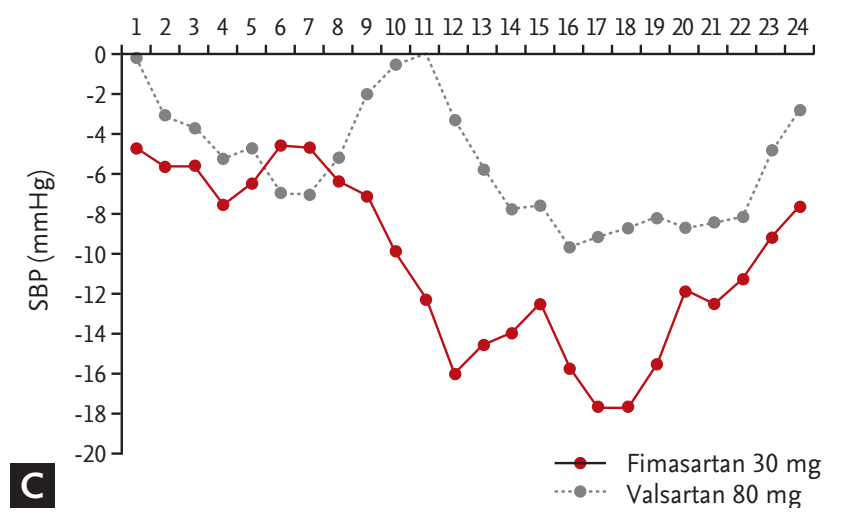

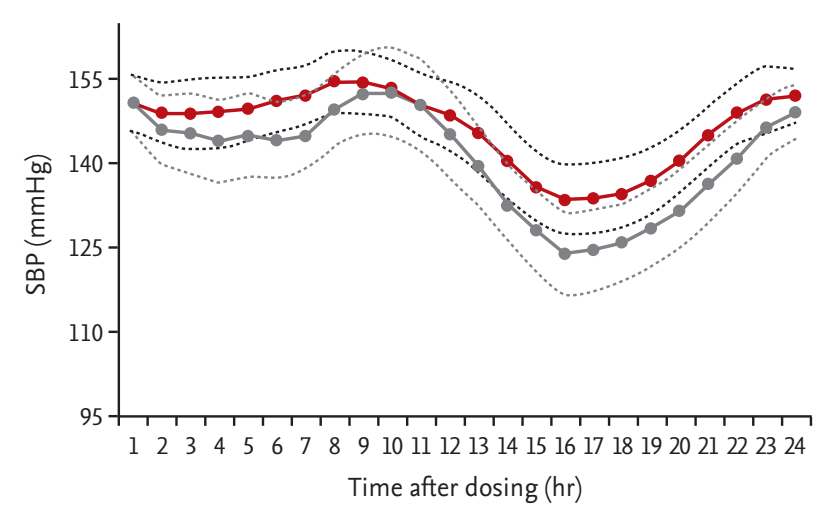

B

......... Baseline, $95 \% \mathrm{Cl}$

$\longrightarrow$ Baseline, mean

Figure 3. 24-Hour systolic blood pressure (SBP) profiles and change from the baseline at week 8. 24-Hour SBP profiles of (A) fimasartan $30 \mathrm{mg}$ and (B) valsartan $30 \mathrm{mg}$ by time after dosing. (C) Change from the baseline at week 8 in 24-hour SBP. Data were analyzed on full analysis set. CI, confidence interval.

Table 2. Change in the mean 24 -hour blood pressures from the baseline at week 8 (full analysis set)

\begin{tabular}{|c|c|c|c|}
\hline Variable & Fimasartan $30 \mathrm{mg}(\mathrm{n}=37)$ & Valsartan $80 \mathrm{mg}(\mathrm{n}=30)$ & $p$ value between groups ${ }^{a}$ \\
\hline \multicolumn{4}{|l|}{ SBP change, mmHg } \\
\hline Baseline & $150.6 \pm 12.1$ & $146.2 \pm 9.2$ & \\
\hline Week 8 & $140.1 \pm 13.0$ & $140.7 \pm 14.6$ & \\
\hline Change from baseline at week 8 & $-10.5 \pm 11.9$ & $-5 \cdot 5 \pm 11.6$ & 0.1392 \\
\hline$p$ value within group ${ }^{\mathrm{b}}$ & $<0.0001$ & 0.0307 & \\
\hline \multicolumn{4}{|l|}{ DBP change, $\mathrm{mmHg}$} \\
\hline Baseline & $93 \cdot 3 \pm 7 \cdot 3$ & $89.7 \pm 5.5$ & \\
\hline Week 8 & $87.4 \pm 8.0$ & $84.7 \pm 7.6$ & \\
\hline Change from baseline at week 8 & $-5.8 \pm 6.4$ & $-5.0 \pm 7.3$ & 0.9732 \\
\hline$p$ value within group ${ }^{\mathrm{b}}$ & $<0.0001$ & 0.0008 & \\
\hline
\end{tabular}

Values are presented as mean $\pm \mathrm{SD}$.

SBP, systolic blood pressure; DBP, diastolic blood pressure.

${ }^{a}$ Difference between treatment groups was analyzed by analysis of covariance .

${ }^{\mathrm{b}}$ Difference within treatment groups was analyzed by paired $t$ test. 
tis (one patient in the fimasartan group, two patients in the valsartan group) was the most commonly reported TEAE. In severity, 10 AEs were mild and three AEs were moderate; influenza (one case, fimasartan), tonsillitis (one case, valsartan), dizziness (one case, valsartan). Drug-related AEs did not occur in either group. And

Table 3. Change in daytime and nighttime SBPs from the baseline at week 8 (full analysis set)

\begin{tabular}{|c|c|c|c|}
\hline Variable & Fimasartan $30 \mathrm{mg}(\mathrm{n}=37)$ & Valsartan $80 \mathrm{mg}(\mathrm{n}=30)$ & $p$ value between groups $\mathrm{s}^{\mathrm{a}}$ \\
\hline \multicolumn{4}{|l|}{ Daytime SBP change, mmHg } \\
\hline Baseline & $154.2 \pm 11.9$ & $151.0 \pm 10.1$ & \\
\hline Week 8 & $146.3 \pm 12.7$ & $146.8 \pm 14.9$ & \\
\hline Change from baseline at week 8 & $-8.0 \pm 12.1$ & $-4.1 \pm 12.1$ & 0.2537 \\
\hline$p$ value within group ${ }^{b}$ & 0.0003 & 0.1442 & \\
\hline \multicolumn{4}{|l|}{ Nighttime SBP change, $\mathrm{mmHg}$} \\
\hline Baseline & $143.5 \pm 14.5$ & $136.6 \pm 13.2$ & \\
\hline Week 8 & $127.8 \pm 16.3$ & $128.6 \pm 16.6$ & \\
\hline Change from baseline at week 8 & $-15 \cdot 7 \pm 14.9$ & $-8.0 \pm 15 \cdot 3$ & 0.1633 \\
\hline$p$ value within group ${ }^{b}$ & $<0.0001$ & 0.0077 & \\
\hline
\end{tabular}

Values are presented as mean $\pm \mathrm{SD}$.

SBP, systolic blood pressure.

${ }^{a}$ Difference between treatment groups was analyzed by analysis of covariance.

${ }^{\mathrm{b}}$ Difference within treatment groups was analyzed by paired $t$ test.
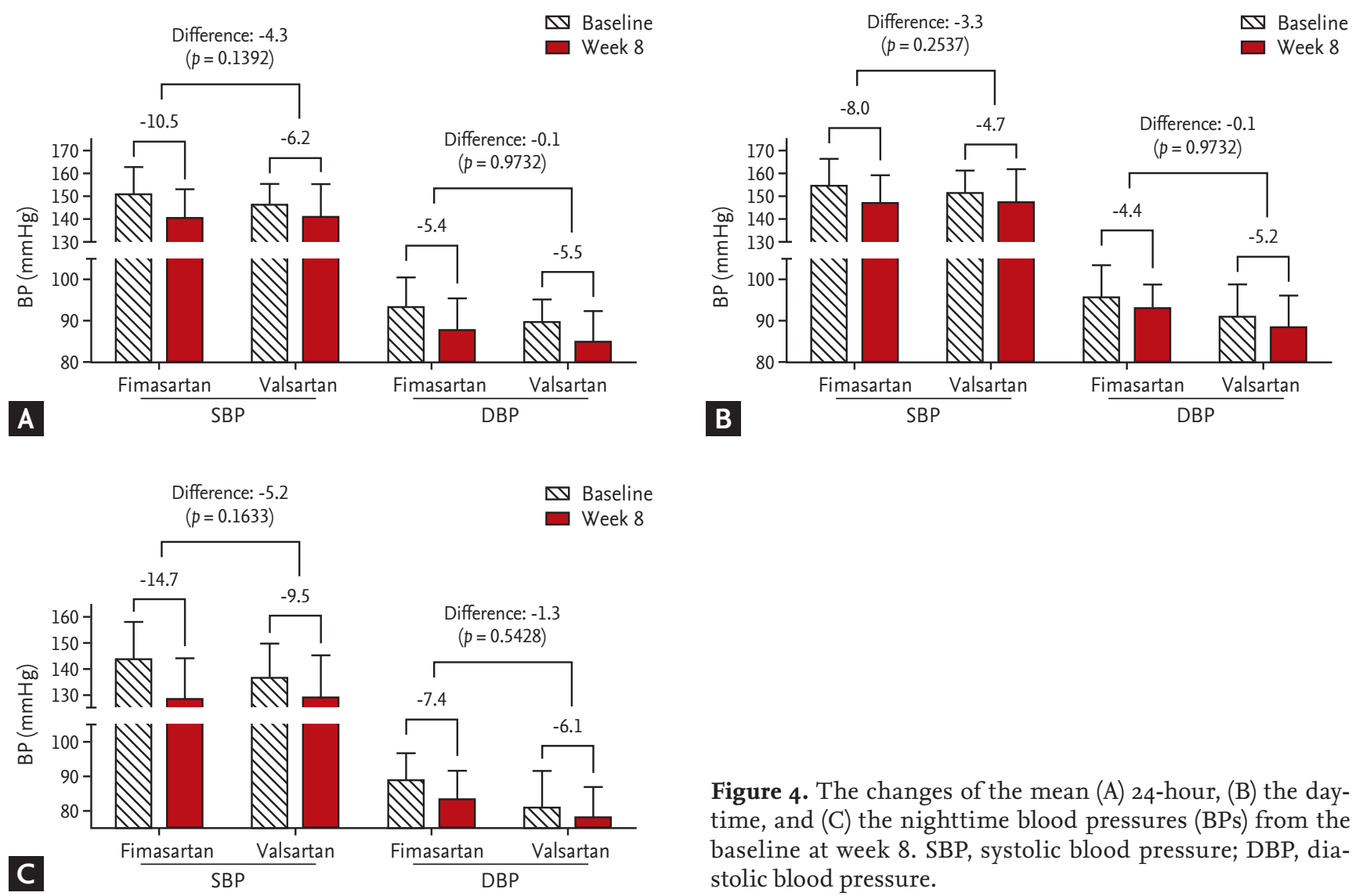

Figure 4. The changes of the mean (A) 24-hour, (B) the daytime, and (C) the nighttime blood pressures (BPs) from the baseline at week 8 . SBP, systolic blood pressure; DBP, diastolic blood pressure. 
Table 4. Trough to peak ratio and smoothness index in SBP and DBP (full analysis set)

\begin{tabular}{|c|c|c|c|}
\hline Variable & Fimasartan $30 \mathrm{mg}(\mathrm{n}=37)$ & Valsartan $80 \mathrm{mg}(\mathrm{n}=30)$ & $p$ value \\
\hline \multicolumn{4}{|c|}{ Trough to peak ratio, median (95\% CI) } \\
\hline SBP (individual) & $0.25(-1.92$ to 0.94$)$ & $-0.03(-1.54$ to 0.64$)$ & $0.3411^{\mathrm{a}}$ \\
\hline SBP (global) & 0.48 & 0.40 & \\
\hline DBP (individual) & $0.23(-0.08$ to 0.26$)$ & 0.18 (0.00 to 0.37$)$ & $0.7288^{a}$ \\
\hline DBP (global) & 0.34 & 0.52 & \\
\hline \multicolumn{4}{|c|}{ Smoothness index, median (range) } \\
\hline SBP & $-0.63(-4.15$ to 1.07$)$ & $-0.29(-3.17$ to 1.21$)$ & $0.2538^{\mathrm{a}}$ \\
\hline DBP & $-0.53(-2.61$ to 0.58$)$ & $-0.54(-2.61$ to 0.89$)$ & $0.7885^{\mathrm{b}}$ \\
\hline
\end{tabular}

SBP, systolic blood pressure; DBP, diastolic blood pressure; CI, confidence interval.

${ }^{a}$ Difference between treatment groups was analyzed by Wilcoxon rank sum test.

bwo sample $t$ test.

there was no case of serious AEs. There was no specific abnormality in laboratory test, vital sign, and physical examination (Table 5).

\section{DISCUSSION}

The objective of the present study was to compare 24hour BP lowering efficacy of low dose (30 $\mathrm{mg}$ ) fimasartan with valsartan $(80 \mathrm{mg})$ in patients with mild to moderate hypertension. The results of this study indicate that the efficacy of low dose (30 mg) fimasartan showed comparable 24-hour BP lowering efficacy with valsartan $(80 \mathrm{mg})$.

In the initial dose finding stage of drug development, fimasartan $20 \mathrm{mg}$ did not show significantly different office BP lowering efficacy compared with the placebo. And a clear demarcation between fimasartan 20 and 60 mg was noted [12]. Therefore, fimasartan $60 \mathrm{mg}$ was chosen as a minimum tablet size. However, in real world situation, half tablet, $30 \mathrm{mg}$ of fimasartan was frequently used as an initial dose or during down-titration step. Therefore, in order to confirm that finding and to prepare the new dosage form, the efficacy study and ABPM study were conducted to evaluate whether low dose 30 $\mathrm{mg}$ ) fimasartan once daily can reduce BP toward the end of the dosing interval and to compare its efficacy with valsartan $80 \mathrm{mg}$. In previous efficacy comparison study of fimasartan $30 \mathrm{mg}$ with valsartan $80 \mathrm{mg}$, after 8 weeks treatment, fimasartan $30 \mathrm{mg}$ showed superior office BP lowering efficacy than valsartan $80 \mathrm{mg}$ in patients with mild to moderate hypertension [26].

ARBs are known to have flat dose-response relation- ship in BP lowering efficacy [32-34]. Previously, fimasar$\tan 60 \mathrm{mg}$ daily was reported to have greater $\mathrm{T} / \mathrm{P}$ ratios and SI values than valsartan $80 \mathrm{mg}$ daily. Longer half-life of fimasartan, 10- to 17.9-hour [19], might explain greater sustainability of 24-hour BP reduction than valsartan which has an intermediate to long half-life of 6 to 10 hours [35,36]. However, increasing the ARB dose was reported to be effective in increasing the duration of the anti-hypertensive effect [37]. Conversely, lower dose of ARBs might have insufficient 24-hour lowering efficacy. Therefore, we could not conclude that lower dose fimasartan had sufficient 24-hour BP lowering efficacy. In this aspect, this study was performed to confirm whether low dose (30 mg) fimasartan maintained 24-hour BP lowering [11]. As expected, T/P ratios and SI values of lower dose fimasartan were both lower than those values reported in fimasartan $60 \mathrm{mg}$ [11]. However, the values were still numerically greater than those of valsartan $80 \mathrm{mg}$.

Although this study did not measure the exact placebo-subtracted $\mathrm{T} / \mathrm{P}$ ratio, $\mathrm{T} / \mathrm{P}$ ratio of fimasartan $30 \mathrm{mg}$ approximated but smaller than 0.5 , which is the lower limit for approval of antihypertensive drugs administered once daily [38], even though recent guideline of the U.S. Food and Drug Administration becomes less strict and flexible [39]. Therefore we should note the possibility of BP fluctuation when prescribing lower dose of fimasartan. And if patients appeal the BP rise in evening or early in the morning, the up-titration of fimasartan dose to increase duration of action will be highly recommended.

The major limitation of this study was the small number of patients and short term duration of study. There- 
Table 5. Key laboratory data change from the baseline at week 8 (full analysis set)

\begin{tabular}{|c|c|c|c|}
\hline Variable & Fimasartan $30 \mathrm{mg}(\mathrm{n}=37)$ & Valsartan $80 \mathrm{mg}(\mathrm{n}=30)$ & $p$ value between groups ${ }^{a}$ \\
\hline $\mathrm{WBC}, 10^{3} / \mu \mathrm{L}$ & & & 0.6959 \\
\hline Baseline & $5.95 \pm 1.26$ & $5.94 \pm 1.28$ & \\
\hline Week 8 & $6.19 \pm 1.76$ & $6.35 \pm 1.57$ & \\
\hline Hemoglobin, g/dL & & & 0.7943 \\
\hline Baseline & $14.7 \pm 1.6$ & $14.8 \pm 1.3$ & \\
\hline Week 8 & $14.9 \pm 1.6$ & $14.7 \pm 1.3$ & \\
\hline Glucose, mg/dL & & & 0.2115 \\
\hline Baseline & $112.7 \pm 30.5$ & $104.2 \pm 15.1$ & \\
\hline Week 8 & $110.0 \pm 26.2$ & $106.5 \pm 17 \cdot 3$ & \\
\hline Cholesterol, mg/dL & & & 0.1264 \\
\hline Baseline & $182 \pm 31$ & $186 \pm 34$ & \\
\hline Week 8 & $188 \pm 32$ & $189 \pm 29$ & \\
\hline Triglyceride, mg/dL & & & 0.5227 \\
\hline Baseline & $137 \pm 58$ & $167 \pm 123$ & \\
\hline Week 8 & $148 \pm 68$ & $155 \pm 117$ & \\
\hline HDL-C, mg/dL & & & 0.8447 \\
\hline Baseline & $51 \pm 11$ & $51 \pm 13$ & \\
\hline Week 8 & $51 \pm 12$ & $53 \pm 13$ & \\
\hline LDL-C, mg/dL & & & 0.0765 \\
\hline Baseline & $115 \pm 29$ & $116 \pm 31$ & \\
\hline Week 8 & $121 \pm 30$ & $116 \pm 27$ & \\
\hline $\mathrm{AST}, \mathrm{U} / \mathrm{L}$ & & & 0.8003 \\
\hline Baseline & $24 \pm 6$ & $24 \pm 7$ & \\
\hline Week 8 & $29 \pm 16$ & $25 \pm 7$ & \\
\hline ALT, U/L & & & 0.9145 \\
\hline Baseline & $24 \pm 10$ & $21 \pm 9$ & \\
\hline Week 8 & $31 \pm 26$ & $22 \pm 10$ & \\
\hline Creatinine, mg/dL & & & 0.6019 \\
\hline Baseline & $0.83 \pm 0.15$ & $0.90 \pm 0.22$ & \\
\hline Week 8 & $0.83 \pm 0.16$ & $0.88 \pm 0.23$ & \\
\hline Uric acid, mg/dL & & & 0.3218 \\
\hline Baseline & $6.0 \pm 1.4$ & $5.9 \pm 1.6$ & \\
\hline Week 8 & $5.9 \pm 1.7$ & $5.6 \pm 1.7$ & \\
\hline
\end{tabular}

Values are presented as mean $\pm \mathrm{SD}$.

WBC, white blood cell; HDL-C, high density lipoprotein cholesterol; LDL-C, low density lipoprotein cholesterol; AST, aspartate aminotransferase; ALT, alanine transferase.

${ }^{a}$ Difference of changes from the baseline at weeks 8 between treatment groups was analyzed by paired $t$ test.

fore we could not confirm any support of superiority of fimasartan $30 \mathrm{mg}$ daily to valsartan $80 \mathrm{mg}$ with statistical significance even though the values were numerically greater than those of valsartan $80 \mathrm{mg}$.

In conclusion, in adult Korean patients who had mild-to-moderate hypertension, the efficacy of low dose (30 mg) fimasartan showed comparable 24-hour BP lowering efficacy compared with valsartan (8o mg). Fimasartan $30 \mathrm{mg}$ effectively maintained BP reduction profile over the full 24-hour dosing interval, suitable for once 
daily dosing. Fimasartan was well tolerated.

\section{KEY MESSAGE}

1. Angiotensin type 1 receptor blockers are known to have flat dose-response relationship in blood pressure (BP) lowering efficacy.

2. The efficacy of low dose (30 mg) fimasartan showed comparable 24-hour BP lowering efficacy with valsartan (8o mg).

3. Fimasartan $30 \mathrm{mg}$ effectively maintained BP reduction profile over the full 24-hour dosing interval, suitable for once daily dosing.

\section{Conflict of interest}

This study was sponsored by Boryung pharmaceutical, Seoul, Korea. The sponsor supported the supply of the investigational products, laboratory test, and clinical research coordinator expenses.

\section{Acknowledgments}

Drs. Byung-Hee Oh and Hae-Young Lee made substantial contributions to the study design. Drs. Byung-Hee $\mathrm{Oh}$ and Hae-Young Lee were contributed in the manuscript writing and figure creation. All the authors were equally contributed in the data collection, data interpretation, literature research, and involved in all stages of manuscript development.

\section{REFERENCES}

1. Ezzati M, Vander Hoorn S, Lawes CM, et al. Rethinking the "diseases of affluence" paradigm: global patterns of nutritional risks in relation to economic development. PLoS Med 2005;2:e133.

2. Neal B, MacMahon S, Chapman N; Blood Pressure Lowering Treatment Trialists' Collaboration. Effects of ACE inhibitors, calcium antagonists, and other blood-pressure-lowering drugs: results of prospectively designed overviews of randomised trials. Blood Pressure Lowering Treatment Trialists' Collaboration. Lancet 2000;356:19551964.

3. Lee HY, Chung WJ, Jeon HK, et al. Impact of the $\beta$-1 adrenergic receptor polymorphism on tolerability and efficacy of bisoprolol therapy in Korean heart failure patients: association between $\beta$ adrenergic receptor polymorphism and bisoprolol therapy in heart failure (ABBA) study. Korean J Intern Med 2016;31:277-287.

4. James PA, Oparil S, Carter BL, et al. 2014 Evidence-based guideline for the management of high blood pressure in adults: report from the panel members appointed to the Eighth Joint National Committee (JNC 8). JAMA 2014;311:507-520.

5. Weber MA, Schiffrin EL, White WB, et al. Clinical practice guidelines for the management of hypertension in the community: a statement by the American Society of Hypertension and the International Society of Hypertension. J Clin Hypertens (Greenwich) 2014;16:14-26.

6. Mancia G, Fagard R, Narkiewicz K, et al. 2013 ESH/ESC guidelines for the management of arterial hypertension: the Task Force for the management of arterial hypertension of the European Society of Hypertension (ESH) and of the European Society of Cardiology (ESC). J Hypertens 2013;31:1281-1357.

7. Lee JH, Bae MH, Yang DH, et al. Angiotensin II type 1 receptor blockers as a first choice in patients with acute myocardial infarction. Korean J Intern Med 2016;31:267276.

8. Choi EK. Angiotensin receptor blocker for stroke prevention in atrial fibrillation: beyond blood pressure lowering? Korean Circ J 2016;46:307-308.

9. Abraham HM, White CM, White WB. The comparative efficacy and safety of the angiotensin receptor blockers in the management of hypertension and other cardiovascular diseases. Drug Saf 2015;38:33-54.

10. Lee HY, Oh BH. Fimasartan: a new angiotensin receptor blocker. Drugs 2016;76:1015-1022.

11. Lee H, Kim KS, Chae SC, Jeong MH, Kim DS, Oh BH. Ambulatory blood pressure response to once-daily fimasartan: an 8-week, multicenter, randomized, double-blind, active-comparator, parallel-group study in Korean patients with mild to moderate essential hypertension. Clin Ther 2013;35:1337-1349.

12. Lee H, Yang HM, Lee HY, et al. Efficacy and tolerability of once-daily oral fimasartan 20 to $240 \mathrm{mg} / \mathrm{d}$ in Korean patients with hypertension: findings from two phase II, randomized, double-blind, placebo-controlled studies. Clin Ther 2012;34:1273-1289.

13. Lee SE, Kim YJ, Lee HY, et al. Efficacy and tolerability of fimasartan, a new angiotensin receptor blocker, com- 
pared with losartan (50/100 mg): a 12-week, phase III, multicenter, prospective, randomized, double-blind, parallel-group, dose escalation clinical trial with an optional 12-week extension phase in adult Korean patients with mild-to-moderate hypertension. Clin Ther 2012;34:552568.

14. Rhee MY, Baek SH, Kim W, et al. Efficacy of fimasartan/ hydrochlorothiazide combination in hypertensive patients inadequately controlled by fimasartan monotherapy. Drug Des Devel Ther 2015;9:2847-2854.

15. Park JB, Sung KC, Kang SM, Cho EJ. Safety and efficacy of fimasartan in patients with arterial hypertension (SafeKanArb study): an open-label observational study. Am J Cardiovasc Drugs 2013;13:47-56.

16. Chang SA, Lim BK, Lee YJ, Hong MK, Choi JO, Jeon ES. A novel angiotensin type I receptor antagonist, fimasartan, prevents doxorubicin-induced cardiotoxicity in rats. J Korean Med Sci 2015;30:559-568.

17. Kim JW, Yi S, Kim TE, et al. Increased systemic exposure of fimasartan, an angiotensin II receptor antagonist, by ketoconazole and rifampicin. J Clin Pharmacol 2013;53:75-81.

18. Lee J, Han S, Jeon S, Hong T, Yim DS. Pharmacokineticpharmacodynamic model of fimasartan applied to predict the influence of a high fat diet on its blood pressurelowering effect in healthy subjects. Eur J Clin Pharmacol 2013;69:11-20.

19. Chi YH, Lee H, Paik SH, et al. Safety, tolerability, pharmacokinetics, and pharmacodynamics of fimasartan following single and repeated oral administration in the fasted and fed states in healthy subjects. Am J Cardiovasc Drugs 2011;11:335-346.

20. Shin KH, Kim TE, Kim SE, et al. The effect of the newly developed angiotensin receptor II antagonist fimasartan on the pharmacokinetics of atorvastatin in relation to $\mathrm{OATP}_{1} \mathrm{~B}_{1}$ in healthy male volunteers. J Cardiovasc Pharmacol 2011;58:492-499.

21. Kim CO, Lee HW, Oh ES, et al. Influence of hepatic dysfunction on the pharmacokinetics and safety of fimasartan. J Cardiovasc Pharmacol 2013;62:524-529.

22. Lee HW, Lim MS, Seong SJ, et al. Effect of age on the pharmacokinetics of fimasartan (BR-A-657). Expert Opin Drug Metab Toxicol 2011;7:1337-1344.

23. Yi S, Kim TE, Yoon SH, et al. Pharmacokinetic interaction of fimasartan, a new angiotensin II receptor antagonist, with amlodipine in healthy volunteers. J Cardiovasc Pharmacol 2011;57:682-689.
24. Jeon H, Lim KS, Shin KH, et al. Assessment of the drugdrug interactions between fimasartan and hydrochlorothiazide in healthy volunteers. J Cardiovasc Pharmacol 2012;59:84-91.

25. Yi S, Kim JW, Kim TE, et al. Effect of multiple doses of fimasartan, an angiotensin II receptor antagonist, on the steady-state pharmacokinetics of digoxin in healthy volunteers. Int J Clin Pharmacol Ther 2011;49:321-327.

26. Youn JC, Ihm SH, Bae JH, et al. Efficacy and safety of $30-$ mg fimasartan for the treatment of patients with mild to moderate hypertension: an 8-week, multicenter, randomized, double-blind, phase III clinical study. Clin Ther 2014;36:1412-1421.

27. Diamant M, Vincent HH. Lisinopril versus enalapril: evaluation of trough:peak ratio by ambulatory blood pressure monitoring. J Hum Hypertens 1999;13:405-412.

28. Staessen JA, Thijs L, Bijttebier G, et al. Determining the trough-to-peak ratio in parallel-group trials: Systolic Hypertension in Europe (SYST-EUR) Trial Investigators. Hypertension 1997;29:659-667.

29. Staessen JA, Bieniaszewski L, Buntinx F, et al. The troughto-peak ratio as an instrument to evaluate antihypertensive drugs: the APTH Investigators. Ambulatory Blood Pressure and Treatment of Hypertension Trial. Hypertension 1995;26:942-949.

30. Coca A, Sobrino J, Soler J, et al. Trough-to-peak ratio, smoothness index, and circadian blood pressure profile after treatment with once-daily fixed combination of losartan 100 and hydrochlorothiazide 25 in essential hypertension. J Cardiovasc Pharmacol 2002;39:824-833.

31. Parati G, Omboni S, Rizzoni D, Agabiti-Rosei E, Mancia G. The smoothness index: a new, reproducible and clinically relevant measure of the homogeneity of the blood pressure reduction with treatment for hypertension. J Hypertens 1998;16:1685-1691.

32. Heran BS, Wong MM, Heran IK, Wright JM. Blood pressure lowering efficacy of angiotensin receptor blockers for primary hypertension. Cochrane Database Syst Rev 2008;(4):CDoo3822.

33. Forclaz A, Maillard M, Nussberger J, Brunner HR, Burnier M. Angiotensin II receptor blockade: is there truly a benefit of adding an ACE inhibitor? Hypertension 2003;41:3136.

34. Jo YI, Na HY, Moon JY, et al. Effect of low-dose valsartan on proteinuria in normotensive immunoglobulin A nephropathy with minimal proteinuria: a randomized trial. 
Korean J Intern Med 2016;31:335-343.

35. Israili ZH. Clinical pharmacokinetics of angiotensin II (AT1) receptor blockers in hypertension. J Hum Hypertens 2000;14 Suppl 1:S73-S86.

36. Taylor AA, Siragy H, Nesbitt S. Angiotensin receptor blockers: pharmacology, efficacy, and safety. J Clin Hypertens (Greenwich) 2011;13:677-686.

37. Morgan T, Griffiths C, Delbridge L. Interaction of ACE inhibitors and $\mathrm{AT}(1)$-receptor blockers on maximum blood pressure response in spontaneous hypertensive rats. J Renin Angiotensin Aldosterone Syst 2002;3:16-18.

38. Rose M, McMahon FG. Some problems with antihypertensive drug studies in the context of the new guidelines. Am J Hypertens 1990;3:151-155.

39. Food and Drug Administration, International Conference on Harmonization (ICH). Draft guidance: E12A principles for clinical evaluation of new antihypertensive drugs [Internet]. Silver Spring (MD): FDA, 2000 [cited 2017 Sep 1]. Available from: http://www.fda.gov/downloads/RegulatoryInformation/Guidances/ucmo73147.pdf. 